\title{
Long-term comparison of the fish community in a Costa Rican rocky shore marine reserve
}

\author{
Mark C. Myers ${ }^{1}$, Jonathan Wagner ${ }^{2} \&$ Christopher Vaughan ${ }^{3,4,5}$ \\ Department of Biology, University of Northern Iowa, 144 McCollum Science Hall, Cedar Falls, IA 50614 USA; \\ mark.myers@uni.edu \\ College of Food, Agriculture, and Natural Sciences, University of Minnesota, St. Paul, MN 55108 USA; \\ wagn0037@umn.edu \\ Department of Forest and Wildlife Ecology, University of Wisconsin-Madison, Madison, WI 53706 USA; \\ cvaughan@wisc.edu \\ International Institute for Wildlife Conservation and Management, Universidad Nacional, Costa Rica \\ Associated Colleges of the Midwest, San Pedro, Costa Rica; cvaughan@acm.edu
}

Received 18-V-2010. Corrected 16-IX-2010. Accepted 13-X-2010.

\begin{abstract}
Despite their role in supporting diverse marine fish communities, tropical rocky shores and reefs have attracted less research and fewer targeted conservation efforts compared to coral reefs. We studied fish community composition in Playa Blanca Marine Reserve $\left(9^{\circ} 40^{\prime} \mathrm{N}-84^{\circ} 40^{\prime} \mathrm{W}\right.$ ), a rocky shore site on the central Pacific coast of Costa Rica. We conducted visual surveys of fishes along six strip transects soon after the area was designated a marine reserve in 1995, then again in 2006 following an eleven-year period of complete protection. We recorded a total of 31406 sightings of 72 species from 30 families. Pomacentrids (42.5\%), labrids $(16.6 \%)$ and haemulids $(14.8 \%)$ dominated the community, accounting for $>70 \%$ of total fish abundance. In comparison to other sites in the region, the fish community was more similar to one reported from Bahia Honda, Panama $\left(7^{\circ} 50^{\prime} \mathrm{N}-81^{\circ} 35 \mathrm{~W}\right)$ than from the geographically more proximate Culebra Bay, Costa Rica $\left(10^{\circ} 45^{\prime}\right.$ $\left.\mathrm{N}-85^{\circ} 43 \mathrm{~W}\right)$. Sixty-one species from 26 families were recorded in 1995; sixty-nine species from 28 families in 2006. Our results suggest that the Playa Blanca Marine Reserve is fulfilling its conservation role. Average fish abundance, species richness and Shannon's index of community diversity were greater in 2006 than 1995 , and fish community composition varied significantly within each transect among years. Much of the change in community composition among years resulted from spatial and temporal variation in the abundance of a few dominant species, including Abudefduf troschelli, Thalassoma lucasanum, Chromis atrilobata, and Stegastes flavilatus/acapulcoensis. Of the 48 species/species groups recorded in both years, 37 (77\%) were more abundant in 2006 than 1995, and several species recorded as uncommon or rare in 1995 were more frequent and abundant in 2006. Fish community composition and the abundance of some species changed in the reserve over time, but further study is needed to determine the role of small-scale marine reserves in the conservation of tropical marine biodiversity. Rev. Biol. Trop. 59 (1): 233-246. Epub 2011 March 01.
\end{abstract}

Key words: diversity, fish assemblage, ichthyofauna, Tropical Eastern Pacific, rocky reef, visual survey, smallscale marine reserves, tropical rocky shores.

The Tropical Eastern Pacific supports a diverse and highly endemic reef fish fauna (Robertson 1998). Because the region's geologic history and variable environmental conditions severely limit coral reef development (Cortés 1997), rocky reefs and shores dominated by algae provide the main habitat for reef-associated fishes throughout much of the Tropical Eastern Pacific. Numerous recent studies have demonstrated that tropical rocky shores support diverse fish communities in the Panamic (Castellanos-Galindo et al. 2005, Dominici-Arosemena et al. 2005, DominiciArosemena \& Wolff 2006), Galapagos (Edgar 
et al. 2004) and Cortez (Alvarez-Filip et al. 2006, Arburto-Oropeza \& Balart 2001) provinces of the Tropical Eastern Pacific, as well as along the tropical Atlantic coast of Brazil (Ferreira et al. 2001, 2004). However, despite their role in supporting diverse fish communities, tropical rocky shores and reefs have attracted relatively less research and far fewer targeted conservation efforts compared to coral reefs (Vroom et al. 2006).

One strategy to conserve tropical marine biodiversity, including rocky shore fish communities, is through the establishment of notake marine reserves (NRC 2001, Sobel \& Dahlgren 2004). Marine protected areas may provide spatial refuge from harvest for adult fish, improve reproduction or recruitment by protecting vulnerable spawning areas or nursery sites, or prevent habitat destruction resulting from human activities. Benefits of marine protected areas, including increased density, biomass, and average body size of target species and increased community diversity relative to unprotected or pre-protection sites, have been well documented in diverse contexts (Halpern \& Warner 2002, Halpern 2003, Lubchenco et al. 2003). In addition, the effects of reserve designation appear to operate independent of reserve size, so that even relatively small marine reserves can contribute to marine conservation goals (Halpern 2003).

We studied fish community composition in a rocky shore marine reserve located at the mouth of the Gulf of Nicoya, Costa Rica in the Panamic province of the Tropical Eastern Pacific. Our objectives were: 1) to document fish community structure present in the subtidal rocky shore habitat at the site and to relate our findings to other studies of fish community structure in the region, and 2) to assess change in fish abundance and community composition at the site following an eleven-year period of marine protection.

\section{MATERIALS AND METHODS}

Study site: The study was carried out in the Punta Leona Biological Reserve on the central Pacific coast of Costa Rica $\left(9^{\circ} 400^{\prime} 41^{\prime \prime}\right.$ - 9 $9^{\circ} 40^{\prime} 44^{\prime \prime} \mathrm{N}$ and $84^{\circ} 39^{\prime} 30^{\prime \prime}$ - 84 $\left.40^{\circ} 15^{\prime \prime} \mathrm{W}\right)$. The reserve includes approximately $3.3 \mathrm{~km}$ of coastline comprised primarily of Mesozoic volcanic and sedimentary rock (CastilloMuñoz 1983). Three small sandy beaches, each $<800 \mathrm{~m}$ in length, are located among the rocky outcroppings in the reserve. In April 1994, the marine environment around the southernmost beach was declared the Playa Blanca Marine Reserve, and a zone of absolute protection was established in the waters extending from the shoreline to a depth of $6 \mathrm{~m}$ at low tide along approximately $1.3 \mathrm{~km}$ of coastline. Since then, commercial and recreational fishing has been prohibited and boat traffic within the reserve has been limited as much as possible by local lifeguards. In March of 2010, Playa Blanca was one of only two beaches nationally to earn five stars in the Costa Rican Tourism Institute's Blue Flag Ecological Program (Programa Bandera Azul Ecológica). The award recognizes the quality of marine resources at the site and the Punta Leona administration's commitment to protection and management of the beach and surrounding marine environment.

Fish surveys: We conducted underwater visual surveys of the fish community in shallow (1.0-3.5m deep) sub-tidal habitat along six strip transects selected to represent the diversity of habitats present in the Playa Blanca Marine Reserve. Transects were $50 \mathrm{~m}$ long $\times$ $10 \mathrm{~m}$ wide. Surveys were conducted during March and April, the last two months of the dry season, in 1995 and 2006. A total of 124 fish surveys were conducted (60 in 1995, 64 in 2006) with each of the six transects surveyed at least ten times in both years.

Visual survey methods were adapted from Williams (1982) and Ferreira et al. (2001). Using snorkeling equipment, a single surveyor recorded the presence and abundance of fishes observed while swimming in a zigzag pattern across the width of each strip transect. To maintain correct transect orientation and width, a 50m tape measure was placed along the centerline and borders were marked with painted 
rocks or flags at $5 \mathrm{~m}$ intervals. In addition, the surveyor actively counted fin kicks and kept visual reference with the transect centerline throughout the survey. Surveyors actively searched for fish under rock outcroppings and within crevices or caves. Surveys averaged $20 \mathrm{~min}$ in duration and were conducted only within two hours of low tide.

Some fishes were difficult to identify to the species level while conducting underwater visual surveys. For morphologically similar species whose identification is based on meristic characteristics (e.g., Diodon holocanthus/hystrix, Kyphosus elegans/analogus and Mugil cephalis/curema), we recorded individuals only to genus-level and conservatively counted species richness as one for each genus. Other species occurred together in large, mixed aggregations (e.g., Haemulon scudderii, maculicauda, steindachneri and flaviguttatum) where the presence of individual species could be verified, but accurately assigning each individual to a species was not feasible. Here we chose to lump species into "species groups" for analysis (Table 1) but included each positively identified species in our counts of species richness. Data on changes in abundance of species within species groups should be interpreted cautiously, as an increase or decrease in abundance of the group does not necessarily imply that all species within the group followed the same trend. However, we believe this approach is conservative and preferable to erroneously generating species-specific results based on inaccurate counts.

We classified each fish species/species group into trophic groups using the categories applied by Dominici-Arosemena \& Wolff (2006). We also modified the mobility categories applied by Floeter et al. (2004) and classified each species/species group as "highly mobile" or "relatively sedentary."

Data analysis: Fish abundance, species richness, Shannon's index of community diversity $\left(\mathrm{H}=-\sum p_{i}\left(\ln p_{i}\right)\right)$ and Simpson's reciprocal index of diversity $\left(\mathrm{D}=1 / \sum\left(n_{i} / N\right)^{2}\right)$ were calculated for each survey $(n=124)$, then averaged for each transect ( $n=6)$ within the two survey years. Paired- $t$ tests were employed to compare the variable means among years using the six transect means as replicates.

We tested for significant change in community composition among years using analysis of similarity (ANOSIM). Community composition varied significantly among transects within years $\left(\mathrm{R}_{1995}=0.6854\right.$ and $\mathrm{R}_{2006}=0.8054$, p-values <0.0001); therefore, we tested for change in community composition among years separately for each of the six transects to remove site effects. Each survey was assigned to an a priori defined group (survey year, 1995 or 2006). ANOSIM is a permutation technique that tests the a priori defined groups against randomly generated groups. The R-statistic generated by ANOSIM is a measure of (dis) similarity of the defined groups, where a value of zero (0) indicates no difference among groups and a value of one (1) indicates that all samples within groups are more similar to one another than to samples in other groups. We conducted ANOSIM using the Bray-Curtis similarity index and 10000 permutations; we report $\mathrm{R}$ statistics and uncorrected $\mathrm{p}$-values for each transect.

We used a variety of techniques to assess which species/species groups were responsible for change in community composition over time. First, we used similarity percentages (SIMPER) analysis to identify which species/species groups made the greatest relative contribution to dissimilarity within transects among years. In addition, after pooling data for all transects, we classified each species/species group as dominant, common, uncommon, or rare in each year (and for the pooled total) by plotting their relative abundance versus frequency of occurrence in surveys. Dominant species had high relative abundance $(>1.5 \%)$ and frequency of occurrence (detected in $>60 \%$ of surveys). Common species relative abundance ranged from 0.20 to $11.12 \%$ and frequency of occurrence from 23 to $68 \%$; uncommon species, $0.08-0.92 \%$ and $5-30 \%$. Species were classified as uncommon or common based on the combination of values and their position 
TABLE 1

Fish species/species groups recorded during surveys of the Punta Leona Marine Reserve during 1995 and 2006

\begin{tabular}{|c|c|c|c|c|c|c|c|c|c|c|c|c|}
\hline \multirow{2}{*}{$\mathrm{ID}^{1}$} & \multirow{2}{*}{ Family } & \multirow{2}{*}{ Species/species group } & \multirow{2}{*}{$\mathrm{Tg}^{2}$} & \multirow{2}{*}{$\mathrm{Mob}^{3}$} & \multicolumn{4}{|c|}{1995} & \multicolumn{4}{|c|}{2006} \\
\hline & & & & & $\mathrm{N}$ & $\mathrm{Ab}^{4}$ & $\mathrm{Fr}^{5}$ & $\mathrm{Cl}^{6}$ & $\mathrm{~N}$ & $\mathrm{Ab}^{4}$ & $\mathrm{Fr}^{5}$ & $\mathrm{Cl}^{6}$ \\
\hline 1 & Acanthuridae & Prionurus laticlavius & $\mathrm{h}$ & $\mathrm{m}$ & 10 & 0.08 & 3 & $\mathrm{r}$ & 62 & 0.33 & 6 & $\mathrm{u}$ \\
\hline 2 & Apogonidae & Apogon dovii & $\mathrm{pl}$ & s & 11 & 0.09 & 3 & $\mathrm{r}$ & 32 & 0.17 & 22 & $\mathrm{u}$ \\
\hline 3 & Balistidae & Balistes polylepis & o & $\mathrm{m}$ & $\mathrm{nr}$ & & & & 6 & 0.03 & 8 & $\mathrm{r}$ \\
\hline 4 & & Pseudobalistes naufragium & o & $\mathrm{m}$ & 61 & 0.47 & 45 & $\mathrm{c}$ & 16 & 0.09 & 21 & $\mathrm{u}$ \\
\hline 5 & & Sufflamen verres & $\mathrm{i}$ & $\mathrm{m}$ & 208 & 1.61 & 85 & $\mathrm{~d}$ & 305 & 1.65 & 84 & d \\
\hline 6 & Belonidae & Tylosurus crocodilus & $\mathrm{p}$ & $\mathrm{m}$ & 9 & 0.07 & 7 & $\mathrm{r}$ & 15 & 0.08 & 2 & $\mathrm{r}$ \\
\hline 7 & Blennidae & Ophoblennius steindachneri & $\mathrm{h}$ & s & 19 & 0.15 & 13 & $\mathrm{u}$ & 28 & 0.15 & 19 & $\mathrm{u}$ \\
\hline 8 & Carangidae & Caranx sexfasciatus or caballus & $\mathrm{p}$ & $\mathrm{m}$ & 625 & 4.85 & 23 & $\mathrm{c}$ & 280 & 1.51 & 19 & $\mathrm{c}$ \\
\hline 9 & & Gnathanodon specious & $\mathrm{p}$ & $\mathrm{m}$ & 23 & 0.18 & 12 & $\mathrm{u}$ & 4 & 0.02 & 5 & r \\
\hline 10 & & Trachinotus rhodopus & $\mathrm{p}$ & $\mathrm{m}$ & $\mathrm{nr}$ & & & & 6 & 0.03 & 3 & $\mathrm{r}$ \\
\hline 11 & Chaetodontidae & Chaetodon humeralis & i & s & 375 & 2.91 & 78 & d & 587 & 3.17 & 78 & d \\
\hline 12 & & Johnrandalia nigrirostris & o & s & 23 & 0.18 & 15 & $\mathrm{u}$ & 29 & 0.16 & 22 & $\mathrm{u}$ \\
\hline 13 & Cirrhitidae & Cirrhitus rivulatus & $\mathrm{c}$ & $\mathrm{s}$ & 8 & 0.06 & 12 & r & 25 & 0.14 & 21 & $\mathrm{u}$ \\
\hline 14 & Dasyatidae & Dasyatis longa & $\mathrm{i}$ & $\mathrm{m}$ & $\mathrm{nr}$ & & & & 1 & 0.01 & 2 & $\mathrm{r}$ \\
\hline 15 & Diodontidae & Diodon sp. & $\mathrm{i}$ & $\mathrm{s}$ & 27 & 0.21 & 27 & $\mathrm{u}$ & 243 & 1.31 & 97 & d \\
\hline 16 & Elopidae & Elops affinis & $\mathrm{p}$ & $\mathrm{m}$ & 43 & 0.33 & 5 & $\mathrm{u}$ & $\mathrm{nr}$ & & & \\
\hline 17 & Fistulariidae & Fistularia commersonii & c & $\mathrm{m}$ & 54 & 0.42 & 22 & $\mathrm{u}$ & 134 & 0.72 & 38 & $\mathrm{c}$ \\
\hline 18 & Gerreidae & Eucinostomus currani & $\mathrm{i}$ & $\mathrm{m}$ & 67 & 0.52 & 17 & $\mathrm{u}$ & 223 & 1.20 & 29 & $\mathrm{c}$ \\
\hline 19 & & Gerres cinereus & i & $\mathrm{m}$ & 4 & 0.03 & 7 & $\mathrm{r}$ & 32 & 0.17 & 11 & $\mathrm{u}$ \\
\hline 20 & Haemulidae & Anistotremus caesius & $\mathrm{c}$ & s & 255 & 1.98 & 60 & $\mathrm{c}$ & 320 & 1.73 & 35 & $\mathrm{c}$ \\
\hline 21 & & Anistotremus taeniatus & $\mathrm{c}$ & s & 156 & 1.21 & 33 & $\mathrm{c}$ & 117 & 0.63 & 41 & $\mathrm{c}$ \\
\hline 22 & & $\begin{array}{l}\text { Haemulon scudderii, maculicauda, } \\
\text { steindachneri or flaviguttatum }\end{array}$ & $\mathrm{c}$ & s & 1068 & 8.28 & 63 & d & 2059 & 11.12 & 37 & $\mathrm{c}$ \\
\hline 23 & Haemulidae & Haemulon sexfasciatum & $\mathrm{c}$ & $\mathrm{s}$ & 246 & 1.91 & 45 & $\mathrm{c}$ & 426 & 2.30 & 49 & $\mathrm{c}$ \\
\hline 24 & Holocentridae & Myripristis leiognathus & $\mathrm{pl}$ & s & 125 & 0.97 & 33 & $\mathrm{c}$ & 181 & 0.98 & 30 & $\mathrm{c}$ \\
\hline 25 & & Sargocentron suborbitalis & $\mathrm{pl}$ & s & 645 & 5.00 & 82 & d & 811 & 4.38 & 83 & d \\
\hline 26 & Kyphosidae & Kyphosus sp. & 0 & $\mathrm{~m}$ & 40 & 0.31 & 33 & $\mathrm{c}$ & 854 & 4.61 & 60 & $\mathrm{c}$ \\
\hline 27 & Labridae & Bodianus diplotaenia & $\mathrm{i}$ & s & 364 & 2.82 & 80 & $\mathrm{~d}$ & 503 & 2.72 & 83 & $\mathrm{~d}$ \\
\hline 28 & & Halichoeres notospilus or nicholsi & $\mathrm{i}$ & s & 408 & 3.16 & 82 & $\mathrm{~d}$ & 217 & 1.17 & 75 & $\mathrm{~d}$ \\
\hline 29 & & Nicholsina denticulata & $\mathrm{h}$ & $\mathrm{m}$ & 22 & 0.17 & 25 & $\mathrm{u}$ & 369 & 1.99 & 56 & $\mathrm{c}$ \\
\hline 30 & & $\begin{array}{l}\text { Scarus rubroviolaceus, perrico, ghobban, } \\
\text { or compressus }\end{array}$ & $\mathrm{h}$ & $\mathrm{m}$ & 312 & 2.42 & 73 & d & 317 & 1.71 & 67 & $\mathrm{c}$ \\
\hline 31 & & Thalassoma lucasanum & $\mathrm{pl}$ & $\mathrm{m}$ & 571 & 4.43 & 78 & $\mathrm{~d}$ & 2141 & 11.57 & 83 & $\mathrm{~d}$ \\
\hline 32 & Lutjanidae & Hoplopagrus guntheri & $\mathrm{c}$ & $\mathrm{s}$ & 8 & 0.06 & 12 & $\mathrm{r}$ & 55 & 0.30 & 57 & c \\
\hline 33 & & Lutjanus argentiventris & $\mathrm{c}$ & $\mathrm{s}$ & 56 & 0.43 & 50 & $\mathrm{c}$ & 44 & 0.24 & 37 & $\mathrm{c}$ \\
\hline 34 & & Lutjanus guttatus & $\mathrm{c}$ & $\mathrm{s}$ & 49 & 0.38 & 17 & $\mathrm{u}$ & 534 & 2.88 & 29 & $\mathrm{c}$ \\
\hline 35 & & Lutjanus inermis & c & $\mathrm{s}$ & $\mathrm{nr}$ & & & & 50 & 0.27 & 10 & $\mathrm{u}$ \\
\hline 36 & & Lutjanus novemfasciatus & $\mathrm{c}$ & $\mathrm{s}$ & $\mathrm{nr}$ & & & & 7 & 0.04 & 10 & $\mathrm{r}$ \\
\hline 37 & Mugilidae & Mugil sp. & $\mathrm{pl}$ & $\mathrm{m}$ & 118 & 0.92 & 10 & $\mathrm{u}$ & 78 & 0.42 & 11 & $\mathrm{u}$ \\
\hline 38 & Mullidae & Mulloidicthyes sp. & $\mathrm{c}$ & $\mathrm{m}$ & 15 & 0.12 & 7 & $\mathrm{u}$ & 23 & 0.12 & 14 & $\mathrm{u}$ \\
\hline 39 & Muraenidae & Gymnomuraena zebra & $\mathrm{c}$ & s & 1 & 0.01 & 2 & $\mathrm{r}$ & 6 & 0.03 & 10 & $\mathrm{r}$ \\
\hline 40 & & Gymnothorax castaneus & $\mathrm{c}$ & $\mathrm{s}$ & 1 & 0.01 & 2 & r & 2 & 0.01 & 3 & r \\
\hline 41 & & Gymnothorax undulatus & $\mathrm{c}$ & $\mathrm{s}$ & $\mathrm{nr}$ & & & & 2 & 0.01 & 3 & $\mathrm{r}$ \\
\hline 42 & & Muraena lentiginosa & $\mathrm{c}$ & $\mathrm{s}$ & 12 & 0.09 & 20 & $\mathrm{u}$ & 15 & 0.08 & 22 & $\mathrm{u}$ \\
\hline
\end{tabular}


TABLE 1 (Continued)

Fish species/species groups recorded during surveys of the Punta Leona Marine Reserve during 1995 and 2006

\begin{tabular}{|c|c|c|c|c|c|c|c|c|c|c|c|c|}
\hline \multirow{2}{*}{$\mathrm{ID}^{1}$} & \multirow{2}{*}{ Family } & \multirow{2}{*}{ Species/species group } & \multirow{2}{*}{$\mathrm{Tg}^{2}$} & \multirow{2}{*}{$\mathrm{Mob}^{3}$} & \multicolumn{4}{|c|}{1995} & \multicolumn{4}{|c|}{2006} \\
\hline & & & & & $\mathrm{N}$ & $\mathrm{Ab}^{4}$ & $\mathrm{Fr}^{5}$ & $\mathrm{Cl}^{6}$ & $\mathrm{~N}$ & $\mathrm{Ab}^{4}$ & $\mathrm{Fr}^{5}$ & $\mathrm{Cl}^{6}$ \\
\hline 43 & Ostraciidae & Ostracion meleagris & $\mathrm{i}$ & $\mathrm{m}$ & $\mathrm{nr}$ & & & & 1 & 0.01 & 2 & $\mathrm{r}$ \\
\hline 44 & Pomacanthidae & Holocanthus passer & 0 & $\mathrm{~s}$ & 42 & 0.33 & 42 & $\mathrm{c}$ & 35 & 0.19 & 25 & $\mathrm{u}$ \\
\hline 45 & & Pomacanthus zonipectus & o & $\mathrm{s}$ & 29 & 0.22 & 32 & $\mathrm{c}$ & 25 & 0.14 & 29 & $\mathrm{u}$ \\
\hline 46 & Pomacentridae & Abudefduf concolor & 0 & $\mathrm{~m}$ & 70 & 0.54 & 22 & $\mathrm{u}$ & 130 & 0.70 & 21 & $\mathrm{c}$ \\
\hline 47 & & Abudefduf troschelii & o & $\mathrm{m}$ & 1749 & 13.57 & 95 & $\mathrm{~d}$ & 2163 & 11.68 & 76 & $\mathrm{~d}$ \\
\hline 48 & & Chromis atrilobata & $\mathrm{pl}$ & $\mathrm{m}$ & 1831 & 14.20 & 62 & $\mathrm{~d}$ & 1073 & 5.80 & 68 & $\mathrm{~d}$ \\
\hline 49 & & Microspathodon bairdi or dorsalis & $\mathrm{h}$ & $\mathrm{s}$ & 350 & 2.71 & 55 & $\mathrm{c}$ & 439 & 2.37 & 51 & $\mathrm{c}$ \\
\hline 50 & & Stagastes flavilatus or acapulcoensis & $\mathrm{h}$ & $\mathrm{s}$ & 2613 & 20.27 & 83 & $\mathrm{~d}$ & 2935 & 15.86 & 84 & d \\
\hline 51 & Scorpaenidae & Scorpaena plumieri mystes & $\mathrm{c}$ & $\mathrm{m}$ & 1 & 0.01 & 2 & $\mathrm{r}$ & 1 & 0.01 & 2 & $\mathrm{r}$ \\
\hline 52 & Serranidae & Cephalopholis panamensis & $\mathrm{c}$ & $\mathrm{s}$ & 26 & 0.20 & 35 & $\mathrm{c}$ & 45 & 0.24 & 43 & $\mathrm{c}$ \\
\hline 53 & & Epinephelus labriformis & $\mathrm{c}$ & s & 58 & 0.45 & 47 & $\mathrm{c}$ & 131 & 0.71 & 68 & $\mathrm{c}$ \\
\hline 54 & & Rypticus bicolor & $\mathrm{c}$ & s & 3 & 0.02 & 5 & $\mathrm{r}$ & 22 & 0.12 & 19 & $\mathrm{u}$ \\
\hline 55 & Sparidae & Calamus brachysomus & $\mathrm{c}$ & $\mathrm{m}$ & $\mathrm{nr}$ & & & & 10 & 0.05 & 8 & r \\
\hline 56 & Stromateidae & Peprilus medius & $\mathrm{c}$ & $\mathrm{m}$ & 22 & 0.17 & 8 & $\mathrm{u}$ & $\mathrm{nr}$ & & & \\
\hline 57 & Tetradontidae & Arothron hispidus & 0 & $\mathrm{~s}$ & 45 & 0.35 & 42 & $\mathrm{c}$ & 56 & 0.30 & 35 & $\mathrm{c}$ \\
\hline 58 & & Arothron meleagris & o & $\mathrm{s}$ & 14 & 0.11 & 18 & $\mathrm{u}$ & 55 & 0.30 & 41 & $\mathrm{c}$ \\
\hline 59 & & Canthigaster punctatissima & o & $\mathrm{s}$ & $\mathrm{nr}$ & & & & 220 & 1.19 & 79 & $\mathrm{~d}$ \\
\hline 60 & & Spheroides lobatus & 0 & $\mathrm{~s}$ & $\mathrm{nr}$ & & & & 6 & 0.03 & 8 & $\mathrm{r}$ \\
\hline 61 & & Sphoeroides annulatus & o & $\mathrm{s}$ & 1 & 0.01 & 2 & $\mathrm{r}$ & 3 & 0.02 & 3 & $\mathrm{r}$ \\
\hline \multirow[t]{2}{*}{62} & Zanclidae & Zanclus cornutus & $\mathrm{i}$ & $\mathrm{s}$ & $\mathrm{nr}$ & & & & 2 & 0.01 & 3 & r \\
\hline & & Total observed & & & 12893 & & & & 18511 & & & \\
\hline
\end{tabular}

1. ID=identification number used to label species/species groups in Fig.1.

2. $\mathbf{T g}$ =trophic group; $\mathbf{c}=$ carnivore, $\mathbf{h}=$ herbivore, $\mathbf{i}=$ invertivore, $\mathbf{o}=$ =mnivore, $\mathbf{p}=$ piscivore, $\mathbf{p l}=$ planktivore

3. Mob=mobility; $\mathbf{m}=$ highly mobile, $\mathbf{s}=$ relatively sedentary

4. $\mathbf{A b}=$ relative abundance expressed as $\%$ of total

5. $\mathbf{F r}=$ frequency of occurrence; $\%$ of surveys in which species/species group was recorded

6. $\mathbf{C l}=$ classification; $\mathbf{d}=$ dominant, $\mathbf{c}=$ common, $\mathbf{u}=$ uncommon, $\mathbf{r}=$ rare.

on the graph. Rare species had low relative abundance $(<0.1 \%)$ and frequency of occurrence $(<12 \%)$. Finally, the abundance of each species/species group was compared among years using the transect means as replicates and paired- $t$ tests or nonparametric Wilcoxon paired-sample tests as appropriate. Analyses were performed using SYSTAT 12.0 (SYSTAT 2010) and PAST (version 2.02) software (Hammer et al. 2001).

\section{RESULTS}

Fish community composition: We recorded a total of 31404 sightings of 72 fish species representing 30 families in the Playa Blanca Marine Reserve (Table 1). Mean abundance of fishes observed per survey was 253.3 $(\mathrm{SD}=165.8)$ with a range from 2 to 954 , while mean species richness per survey was 18.8 (6.7) and ranged from 2 to 31 species.

Using the data pooled for both years, we classified each species/species group as dominant, common, uncommon or rare according to its relative abundance and frequency of occurrence (Fig. 1a). Eleven dominant species/ species groups (Abudefduf troschelii, Bodianus diplotaenia, Chromis atrilobata, Chaetodon humeralis, Haemulon scudderii/maculicaudal steindachneri/flaviguttatum, Halichoeres 

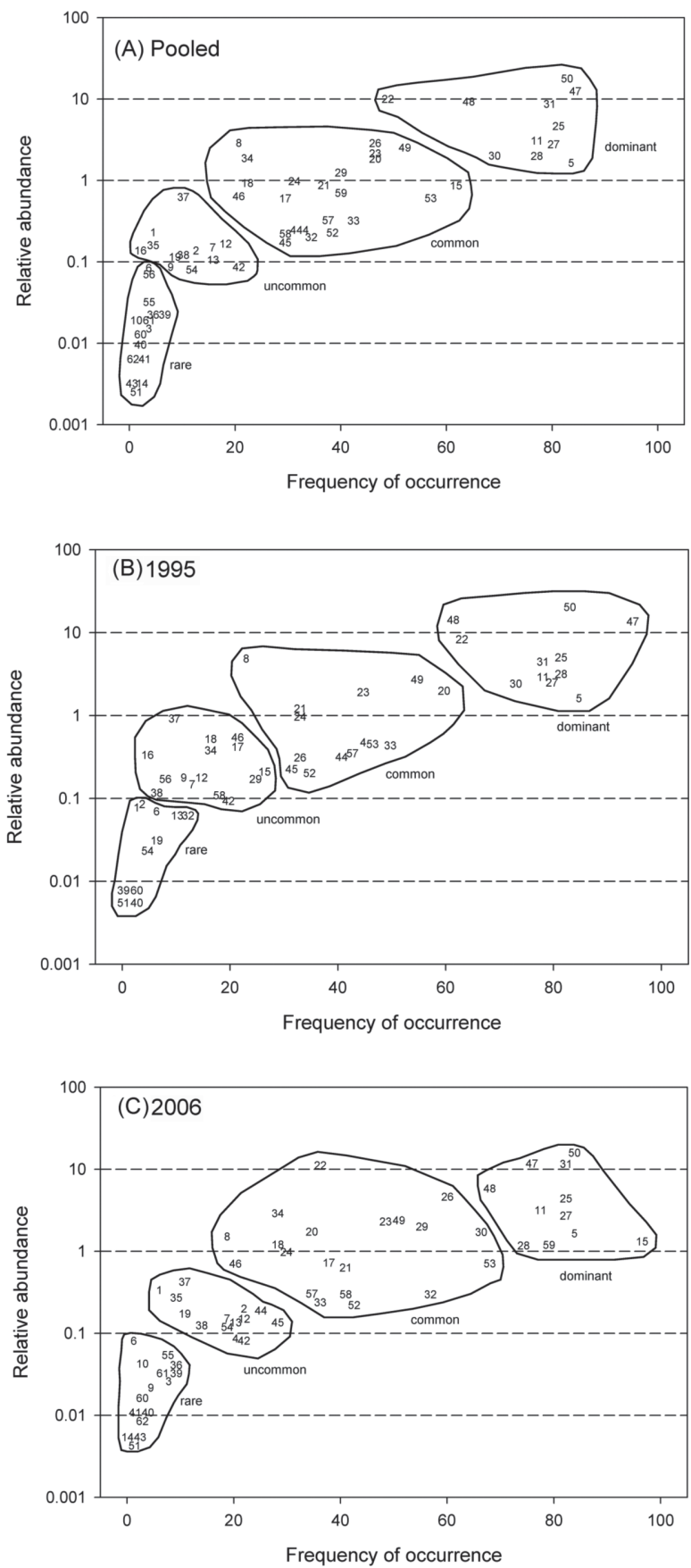

Fig. 1. Plot of relative abundance versus frequency of occurrence for 62 fish species/species groups in the Playa Blanca Marine Reserve, Costa Rica (A) pooled for the entire study period (B) in 1995 and (C) in 2006. Species/species groups were plotted using the values from the "ID" column in Table 1 as labels. Species/species groups were classified as dominant, common, uncommon, or rare based on their position on the graph. 
notospilus/nicholsi, Sargocentron suborbitalis, Scarus rubroviolaceus/perrico/ghobban/compressus, Stegastes flavilatum/acapulcoensis, Sufflamen verres and Thalassoma lucasanum) accounted for $74.1 \%$ of total fish abundance. At the family level, Pomacentridae was most dominant, accounting for $42.5 \%$ of all individuals, with Labridae (16.6\%) and Haemulidae $(14.8 \%)$ the next highest ranking families.

Twenty-three common species/species groups were characterized by moderate abundance and frequency of occurrence. Some common species (e.g., Caranx sexfasciatus/ caballus, Lutjanus guttatus and Kyphosus sp.) were encountered less frequently but in large aggregations, while others were encountered in a high percentage of all surveys but as solitary individuals (e.g., species in Diodontidae, Fistulariidae, Serranidae and Tetradontidae). Common species accounted for $23.4 \%$ of total fish abundance.

Twenty-eight species/species groups were classified as uncommon or rare. Fifteen uncommon species had relative abundances from $0.08 \%$ to $0.62 \%$ and frequency of occurrence $\leq 21 \%$. Uncommon species accounted for $2.2 \%$ of total fish abundance. Thirteen rare species had both low relative abundance $(<0.1 \%)$ and frequency of occurrence $(<12 \%)$ and accounted for just $0.3 \%$ of total abundance. The rarest families were Dasyatidae, Scorpenidae, Zanclidae and Ostraciidae, each represented by a single species accounting for $<0.01 \%$ of total abundance.

In terms of trophic groups, nearly one-third of fish species were carnivores (25 species, $19.2 \%$ total fish abundance), yet herbivores (11 species) accounted for the greatest proportion of total fish abundance (23.8\%). Overall, the distribution of species among trophic groups was quite equitable: omnivores $(19.4 \%, 13$ species), invertivores (18.8\%, 11 species), planktivores $(15.6 \%, 6$ species) and piscivores $(3.2 \%$, 6 species).

Community change over time: We recorded 12893 sightings of 61 species from 26 families in 1995 and 18511 sightings of 69 species from 28 families in 2006. Fish abundance (paired $t$-test, $\mathrm{df}=5, t=-2.575, \mathrm{p}=0.050$ ) and species richness (paired $t$-test, $\mathrm{df}=5$, $t=-2.571, \mathrm{p}=0.050)$ were significantly greater in 2006 than 1995. Shannon's index of diversity, which is sensitive to changes in rare species, was significantly greater in 2006 (paired $t$-test, $\mathrm{df}=5, \mathrm{t}=-2.624, \mathrm{p}=0.047$ ), but there was no difference in Simpson's index of diversity, which is sensitive to changes in the most abundant species among years (paired $t$-test, $\mathrm{df}=5$, $\mathrm{t}=-1.873, \mathrm{p}=0.120$ ).

We detected significant change in fish community composition (all $\mathrm{p}<0.01$ ) among years in each of the six transects $\left(\mathrm{R}_{\mathrm{T} 1}=0.2434\right.$; $\mathrm{R}_{\mathrm{T} 2}=0.7153 ; \quad \mathrm{R}_{\mathrm{T} 3}=0.7078 ; \quad \mathrm{R}_{\mathrm{T} 4}=0.6967$; $\mathrm{R}_{\mathrm{T} 5}=0.8635 ; \mathrm{R}_{\mathrm{T} 6}=0.7292$ ). The five species/ species groups that contributed most to community dissimilarity among years within each transect are listed in Table 2. These species/ species groups accounted for 47.7 to $84.4 \%$ of the dissimilarity among years within transects. Much of the variation in community composition among years resulted from variation in the abundance of a few dominant species. For example, Abudefduf troschelli was identified as an influential species in all six transects; Thalassoma lucasanum and Chromis atrilobata in five; and Stegastes flavilatus/acapulcoensis in four. These were among the most abundant and omnipresent species in the study, but their average abundance varied substantially among transects and years. Not all species identified as influential by SIMPER were dominant; some, including Lutjanus guttatus, Eucinostomus currani and Nicholsina denticulate, were uncommon in 1995 but were more frequent and abundant and classified as common in 2006.

We also assessed change in community composition by comparing individual species/ species group classifications among years. Thirteen of the 19 species whose classification changed among years were more frequent and abundant in 2006 (Table 1; Fig. 1b and 1c). Six species classified as rare in 1995 were uncommon (Prionurus laticlavius, Apogon dovii, Cirrhitus rivulatus, Gerres cinereus and Rypticus bicolor) or common (Hoplopagrus guntheri) in 
TABLE 2

Fish species/species groups most responsible for dissimilarity in community composition among years in each transect

\begin{tabular}{|c|c|c|c|c|c|c|}
\hline $\operatorname{Tr}^{1}$ & Dis $^{2}$ & Species/species group & $\mathrm{Ab}_{1995}{ }^{3}$ & $\mathrm{Ab}_{2006}{ }^{3}$ & $\mathrm{Co} \%{ }^{4}$ & $\mathrm{Cu} \%^{5}$ \\
\hline \multirow[t]{5}{*}{1} & 42.75 & Abudefduf troschelii & 77.2 & 78.6 & 12.76 & 29.86 \\
\hline & & Thalassoma lucasanum & 9.2 & 30.6 & 4.849 & 41.2 \\
\hline & & Stegastes flavilatus or acapulcoensis & 57.6 & 69.7 & 3.845 & 50.2 \\
\hline & & Microspathodon bairdi or dorsalis & 4.7 & 16 & 2.473 & 55.98 \\
\hline & & Chromis atrilobata & 9.7 & 4.73 & 1.997 & 60.65 \\
\hline \multirow[t]{5}{*}{2} & 48.34 & Chromis atrilobata & 98.3 & 26.1 & 7.356 & 15.22 \\
\hline & & Haemulon scudderii, maculicauda, steindachneri, or flaviguttatum & 60.9 & 132 & 6.284 & 28.22 \\
\hline & & Kyphosus sp. & 1 & 69.1 & 6.278 & 41.2 \\
\hline & & Abudefduf troschelii & 39.8 & 74.9 & 4.04 & 49.56 \\
\hline & & Thalassoma lucasanum & 11.1 & 52.4 & 3.936 & 57.7 \\
\hline \multirow[t]{5}{*}{3} & 86.87 & Haemulon scudderii, maculicauda, steindachneri, or flaviguttatum & 0 & 66.3 & 35.57 & 40.95 \\
\hline & & Lutjanus guttatus & 4.3 & 39.3 & 20.27 & 64.29 \\
\hline & & Eucinostomus currani & 5.8 & 18 & 10.43 & 76.29 \\
\hline & & Mugil sp. & 7 & 0 & 4.102 & 81.01 \\
\hline & & Abudefduf troschelii & 4.3 & 0 & 2.954 & 84.41 \\
\hline \multirow[t]{5}{*}{4} & 57.1 & Stegastes flavilatus or acapulcoensis & 31.4 & 64.5 & 8.397 & 14.55 \\
\hline & & Thalassoma lucasanum & 9.4 & 33.8 & 6.004 & 24.96 \\
\hline & & Chromis atrilobata & 23.7 & 10.1 & 4.675 & 33.06 \\
\hline & & Abudefduf troschelii & 25.5 & 20 & 4.331 & 40.56 \\
\hline & & Sargocentron suborbitalis & 4.4 & 21.1 & 4.091 & 47.65 \\
\hline \multirow[t]{5}{*}{5} & 56.1 & Thalassoma lucasanum & 10.3 & 49.5 & 13.05 & 23.26 \\
\hline & & Stegastes flavilatus or acapulcoensis & 30.3 & 52.1 & 7.464 & 36.57 \\
\hline & & Nicholsina denticulata & 0.5 & 15.2 & 4.934 & 45.36 \\
\hline & & Abudefduf troschelii & 6.7 & 11.9 & 3.824 & 52.18 \\
\hline & & Chromis atrilobata & 3.2 & 9.64 & 3.235 & 57.95 \\
\hline \multirow[t]{5}{*}{6} & 43.72 & Haemulon scudderii, maculicauda, steindachneri, or flaviguttatum & 39.3 & 0 & 6.399 & 14.64 \\
\hline & & Chromis atrilobata & 48.2 & 54.3 & 6.015 & 28.39 \\
\hline & & Stegastes flavilatus or acapulcoensis & 73.9 & 47.8 & 4.116 & 37.81 \\
\hline & & Thalassoma lucasanum & 17.1 & 36.3 & 3.592 & 46.02 \\
\hline & & Abudefduf troschelii & 21.4 & 19.8 & 3.104 & 53.12 \\
\hline
\end{tabular}

1. $\mathbf{T r}=$ transect

2. Dis=average dissimilarity

3. $\mathbf{A b}=$ mean abundance

4. $\mathbf{C o} \%=\%$ of Dis accounted for by species/species group

5. $\mathbf{C u} \%=$ cumulative $\%$ contribution of listed species/species groups to Dis.

2006. Six uncommon species (Fistularia commersonii, Eucinostomus currani, Nicholsina denticulate, Lutjanus guttatus, Abudefduf concolor and Arothron meleagris) were classified as common, and Diodon sp. was uncommon in 1995 but dominant in 2006. Six species/species groups were less frequent and abundant in 2006, including Gnathanodon specious (uncommon to rare); Pseudobalistes naufragium, Holocanthus passer and Pomacanthus zonipectus (common to uncommon); Наетиlon scudderii/maculicauda, steindachneri/flaviguttatum and Scarus rubroviolaceus/perrico/ ghobban/compressus (dominant to common). 
We also directly compared the relative abundance of the 48 species/species groups that were recorded in both years, independent of their frequency of occurrence. Of these, thirty-seven (77\%) were more abundant in 2006 and only $11(23 \%)$ were more abundant in 1995 (Table 1). The abundance of seven species/species groups varied significantly by year. Of these, six (Diodon sp., Epinephelus labriformis, Hoplopagrus guntheri, Kyphosus sp., Nicholsina denticulate and Thalassoma lucasanum) were more abundant in 2006 than 1995 (Table 3). Only Halichoeres notospilus/ nicholsi were more abundant in 1995 than 2006. At the family level, serranids (Wilcoxon paired sample test, $\mathrm{df}=5, z=2.201, \mathrm{p}=0.028$ ) were significantly more abundant in 2006 . We found no significant difference in the abundance of fishes among years when pooled by trophic groups or mobility classes.

Finally, the presence/absence of less abundant species also affected community composition over time. Fourteen of 62 species/species groups were recorded in one year but not the other (Table 1). Eleven of these were recorded in 2006 but not 1995, including Balistes polylepis, Calamus brachysomus, Canthigaster punctatissima, Dasyatis longa, Gymnothorax undulatus, Lutjanus inermis, Lutjanus novemfasciatus, Ostracion meleagris, Spheroides lobatus,
Trachinotus rhodopus and Zanclus cornutus. All of these were rare except for Lutjanus inermis, which was classified as uncommon and occurred in moderate numbers in $<10 \%$ of surveys in 2006, and Canthigaster punctatissima, which was significantly more abundant (Table 3), occurred in $80 \%$ of surveys, and was classified as dominant in 2006. Two uncommon species (Elops affinis and Peprilus medius) recorded in 1995 were absent in 2006.

\section{DISCUSSION}

Community composition: The Playa Blanca Marine Reserve supports a diverse fish community similar in species composition to other reported sites in the Panamic Province of the Tropical Eastern Pacific (Table 4). Dominici-Arosemana \& Wolff (2006) documented 126 species from 44 families in Bahia Honda, Gulf of Chiriquí, Panama ( $7^{\circ} 50^{\prime} \mathrm{N}-81^{\circ} 35 \mathrm{~W}$ ), the most diverse Tropical Eastern Pacific reef fish community reported to date. However, Dominici-Arosemena \& Wolff (2006) sampled 48 transects over a larger geographic area and used SCUBA to sample to depths up to $15 \mathrm{~m}$, while we used snorkeling to sample six transects at a localized site at depths $<3.5 \mathrm{~m}$. As a consequence of this variation in sampling effort and methods, total diversity at Playa Blanca

TABLE 3

Species/species groups with significant differences in abundance among years

\begin{tabular}{llcccc}
\multicolumn{1}{c}{ Family } & \multicolumn{1}{c}{ Species/Species group } & $\mathrm{Ab}^{1}{ }_{1995}$ & $\mathrm{Ab}_{2006}$ & $\mathrm{Test}_{\text {statistic }}{ }^{2}$ & $\mathrm{p}$ \\
Diodontidae & Diodon sp. & 1.017 & 3.786 & $t=-4.778$ & 0.028 \\
Kyphosidae & Kyphosus sp. & 0.750 & 3.572 & $z=2.023$ & 0.043 \\
Labridae & Halichoeres notospilus or nicholsi & 6.800 & 3.489 & $t=4.105$ & 0.009 \\
& Nicholsina denticulata & 0.100 & 1.950 & $z=2.023$ & 0.043 \\
& Thalassoma lucasanum & 9.517 & 33.489 & $t=-4.141$ & 0.009 \\
Lutjanidae & Hoplopagrus guntheri & 0.133 & 0.852 & $t=-5.711$ & 0.002 \\
Serranidae & Epinephelus labriformis & 0.967 & 2.069 & $t=-2.549$ & 0.050 \\
Tetradontidae & Canthigaster punctatissima & 0.000 & 4.014 & $t=-3.907$ & 0.011
\end{tabular}

1. $\mathbf{A} \mathbf{b}=$ abundance. If $t$-statistic is presented, means are reported; if $z$-statistic, medians.

2. Where the differences between paired abundance values were normally distributed, we employed paired- $t$ tests and report $t$-statistics. Where differences were non-normal, nonparametric Wilcoxon paired-sample tests were applied and $z$-statistics are reported. 
TABLE 4

Comparison of number of fish species and families reported from various Tropical Eastern Pacific sites

\begin{tabular}{|c|c|c|c|c|c|}
\hline Authors & Site & Species & Families & $\begin{array}{l}\text { Shared } \\
\text { species }^{1}\end{array}$ & $\begin{array}{c}\text { Shared } \\
\text { families }^{2}\end{array}$ \\
\hline Current study & $\begin{array}{l}\text { Playa Blanca Marine Reserve, Gulf of } \\
\text { Nicoya, Costa Rica }\end{array}$ & 72 & 30 & & \\
\hline $\begin{array}{l}\text { Dominici-Arosemena \& } \\
\text { Wolff (2006) }\end{array}$ & Bahia Honda, Gulf of Chiriqui, Panama & 126 & 44 & $64(89 \%)$ & $27(90 \%)$ \\
\hline $\begin{array}{l}\text { Dominici-Arosemena et } \\
\text { al. }(2005)\end{array}$ & Culebra Bay, Gulf of Papagayo, Costa Rica & 75 & 28 & $51(71 \%)$ & $20(67 \%)$ \\
\hline $\begin{array}{l}\text { Alvarez-Filip et al. } \\
\text { (2006) }\end{array}$ & $\begin{array}{l}\text { Cabo Pulmo Reef, Gulf of California, } \\
\text { Mexico }\end{array}$ & 62 & 23 & $41(57 \%)$ & $20(67 \%)$ \\
\hline Arbuto-Oropeza \& Balart & Los Islotes, Gulf of California, Mexico & 74 & 28 & $41(57 \%)$ & $21(70 \%)$ \\
\hline
\end{tabular}
(2001)

1. Number and percentage of species recorded in the Playa Blanca Marine Reserve also recorded in the study indicated.

2. Number and percentage of families recorded in the Playa Blanca Marine Reserve also recorded in the study indicated.

was predictably lower; however, species composition between the two sites was relatively similar. Nearly $90 \%$ of the families and species recorded in our study were also reported by Dominici-Arosemena \& Wolff (2006) (Table 4). The four families recorded at Playa Blanca (Belonidae, Elopidae, Sparidae and Stromateidae) absent from Bahia Honda were each represented by a single rare species.

Seventeen families reported by DominiciArosemena \& Wolff (2006) from Bahia Honda were absent from the Playa Blanca Marine Reserve. Twelve of the 17 families were represented by a single species and none by more than three species. One of the most significant differences in community composition between the two sites was the underrepresentation of small-bodied, cryptic benthic fishes in our study. We believe this is a result of our sampling methods; we used relatively wide transects and did not use SCUBA equipment. Future studies employing SCUBA to more intensively sample benthic fishes along narrower transects would likely detect chaenopsids, gobiids, labrisomids, opistognathids, ophichthids and/or tripterygiids that would increase estimates of species richness in the Playa Blanca Marine Reserve.

Additionally, species richness of some families common to both sites was higher in Bahia Honda. For example, the species richness of carnivorous and piscivorous serranids (Bahia Honda: 10 species, Playa Blanca: 3), carangids (BH: 11, PB: 4) and lutjanids (BH: 8, PB: 5) were greater in Bahia Honda compared to Playa Blanca. Many of the species absent from Playa Blanca were reported from just one or a few of the 12 sites in DominiciAreosemena \& Wolff's (2006) study, and many of those sites were at depths $>3.5 \mathrm{~m}$. Labridae (BH: 10, PB: 4) and Acanthuridae (BH: 5, PB: 1) species richness was also lower in Playa Blanca.

Interestingly, while approximately $90 \%$ of families and species from Playa Blanca were also reported from Bahia Honda, Panama, the species composition of Playa Blanca was less similar to that of Culebra Bay, Gulf of Papagayo, Costa Rica $\left(10^{\circ} 45^{\prime} \mathrm{N}-85^{\circ} 43 \mathrm{~W}\right)$, even though this site is closer geographically and had similar measures of total diversity (Table 4). Sixtyseven percent of families and $71 \%$ of species from Playa Blanca were reported from Culebra Bay (Dominici-Arosemana et al. 2005). Future studies using standardized survey methods at multiple sites may elucidate patterns of variation in species composition along this latitudinal gradient (Ferriera et al. 2004).

In comparison to sites in the Cortez Province of the Tropical Eastern Pacific, measures of species richness were comparable; however, 
community composition, especially at the species level, was less similar compared to other Panamic Province sites (Table 4). For example, while nearly $70 \%$ of the families recorded in our study were also recorded at sites in the Gulf of California, only about $57 \%$ of species were (Alvarez-Filip et al. 2006, Aburto-Oropeza $\&$ Balart 2001). Further, only $61 \%$ of species classified as "dominant" or "frequent and abundant" by Aburto-Oropeza \& Balart (2001) at Los Islotes, Gulf of California were present in the Playa Blanca Marine Reserve.

While there was significant variation in species composition across Tropical Eastern Pacific sites, a common feature of reef fish communities in the region appears to be the dominance of pomacentrids, labrids, and haemulids. These families collectively accounted for $73.9 \%$ of total fish abundance in the Playa Blanca Marine Reserve and were among the most dominant families in Bahia Honda (Dominici-Arosemena \& Wolff 2006), Culebra Bay (Dominici-Arosemena et al. 2005) and the Cabo Pulmo Reef (Alvarez-Filip et al. 2006). Pomacentrids, particularly Stegastes acapulcoensis and S. flavilatus, were ubiquitously observed throughout Playa Blanca Marine Reserve. They were especially prevalent where broken rock covered by dense brown algae (Padina and Sargassum) bordered sandy substrate. This combination of physical and biological substrate characteristics provided ideal habitat for territorial herbivores like Stegastes. Other abundant pomacentrids included Abudefduf troschelii and Chromis atrilobata, which appeared to partition food resources based on depth. Abudefduf troschelii, an omnivore, fed on plankton and invertebrates primarily in the shallowest parts of each transect, while C. atrilobata, a planktivore, occurred in greatest abundance in the upper half of the water column in the deeper, offshore sections of the transects.

The dominance of the labrids $(16.6 \%$ total fish abundance) was primarily a function of Thalassoma lucasanum, a planktivorous feeder found with high frequency at consistently high abundance in all non-sand transects. The haemulids accounted for $14.8 \%$ of total fish abundance in our study. Sixty-two percent of total haemulid abundance was observed in a single transect where very large schools of juveniles congregated in large rock caves and overhangs. Given their high relative abundance, the haemulid's frequency of occurrence was relatively low ( $<40 \%$ in 2006$)$, indicating that these species likely move in and out of the Playa Blanca Marine Reserve frequently.

Change in community composition and marine reserve management: The Playa Blanca Marine Reserve appears to be fulfilling its conservation role. Most measures of fish abundance, species richness, and diversity were greater in 2006 (after 11 years of protection) compared to 1995 (1 year after reserve designation). Seventy-seven percent of species species/ species groups reported in both years were more abundant in 2006, and seven of eight species/species groups with significant differences in mean abundance among years were more abundant in 2006. Thirteen of 19 species/ species groups whose classification changed among years were more frequent and abundant in 2006. While we lack data from unprotected control sites over the same time period, we believe that the limitations on fish harvest and habitat degradation conferred by the site's protected status have promoted an increase in fish abundance and diversity. Extraction of ornamental fishes such as T. lucasanum for the aquarium trade was common at Playa Blanca in the early-to mid-1990's (C. Vaughan 2010, pers. observ.). Creation of the marine reserve in 1994 and subsequent enforcement of regulations effectively halted this activity and probably contributed to the increase in $T$. lucasanum and other species observed in 2006.

We had hypothesized that species/species groups at higher trophic levels and/or species classified as relatively sedentary might show a greater response to protection; however, we found no significant difference in the abundance of fishes among years when pooled by trophic groups or mobility classes. Instead, consistent with the results of Halpern (2003), 
the increase in fish abundance was distributed proportionally among trophic groups.

The Gulf of Nicoya is an important nursery area for many fishes (Campos et al. 1984), and our observations suggest that within the Gulf, small protected areas such as the Playa Blanca Marine Reserve can contribute to fish conservation in the region. For example, we observed a substantial increase in the abundance of juvenile lutjanids and serranids in the reserve in 2006 compared to 1995 , and lutjanid species richness increased from three species in 1995 to five in 2006. Both lutjanids and serranids are relatively sedentary carnivores, and their increased abundance could be a result of reduced harvest and/or increased food availability in the reserve after protection. In 2006, large adult $L$. novemfasciatus, a species absent in 1995, were observed among the rock outcrops and overhangs of the deeper transects, and small schools of L. inermis were frequently observed along vertical rock walls. Future studies and continued monitoring are needed to assess the effects of protection in the long-term; Micheli et al. (2004) found that the magnitude of positive response of piscivorous fishes to protection increased substantially after $>10 \mathrm{yrs}$ of protection. The potential role of the Playa Blanca Marine Reserve as a breeding and maturation zone for commercially exploited fishes and as a source of emigrants to adjacent fishing grounds also warrants further investigation.

Marine reserves may also promote habitat diversity by reducing human disturbance and habitat alteration. The transect with the greatest dissimilarity in fish composition among years (see Table 2, transect 3) was one characterized by the highest percentage of sandy substrate. Within this transect, seagrass coverage increased from $<5 \%$ in 1995 to approximately $20 \%$ in 2006. Fish response to the change in habitat characteristics was apparent; average fish abundance increased five-fold and average species richness doubled in 2006 compared to 1995. In addition, species composition between years was quite different. While planktivores and mobile invertebrate feeders like Mugil sp. and Eucinostomus currani were most abundant in 1995, in 2006 the transect was dominated by schools of juvenile carnivores (especially Haemulon maculicauda, Haemulon steindachneri, and Lutjanus guttatus) actively searching for prey within the seagrass.

In conclusion, we have documented significant increases in fish abundance, species richness, and diversity and significant change in community composition in the Playa Blanca Marine Reserve following eleven years of protection. Future studies incorporating unprotected controls and more frequent monitoring are needed to further elucidate the contribution of small marine reserves to regional conservation goals.

\section{ACKNOWLEDGMENTS}

We are grateful to many individuals who provided expertise, information, and logistical support over the course of this study. Eugenio Gordienko and Miguel Fernandez allowed access to Punta Leona facilities and the Playa Blanca Marine Reserve. Memo Hernandez and Andrea Osorio provided logistical support on site. Fieldwork was supported by the Associated Colleges of the Midwest (Spring Semester Research Program in Costa Rica) and Sigma Xi. Institutional support was provided by the University of Northern Iowa for M. Myers and the Universidad Nacional for C. Vaughan. We thank Sarah Faust for her assistance with data curation and literature review and three anonymous reviewers for their insightful comments on this manuscript.

\section{RESUMEN}

Los corales y los bordes rocosos tropicales están compuestos por diversas comunidades de peces pero han atraído menos investigación o conservación que los arrecifes coralinos. Estudiamos la composición de una comunidad de peces en la Reserva Marina Playa Blanca, un borde rocoso en la costa del Pacífico central de Costa Rica. Realizamos muestreos visuales de peces en seis transectos inmediatamente después que el área fue designada como reserva marina en 1995, y de nuevo en 2006, después de once años de protección. Observamos un total de 31406 avistamientos de 72 especies y 30 familias. Los pomacentridos $(42.5 \%)$, labridos $(16.6 \%)$ y haemulidos (14.8\%) dominaron la comunidad, sumando $>70 \%$ de la 
abundancia total de peces. Nuestros resultados sugieren que la Reserva Marina Playa Blanca está cumpliendo con su papel de conservación. El promedio de abundancia, riqueza y diversidad de peces fueron mayores en 2006 comparados con 1995, y muchas especies clasificadas como no comunes o raras en 1995 fueron más abundantes y observadas más frecuentemente en 2006. La composición de la comunidad cambió significativamente entre 1995 y 2006, la mayoría del cambio debido a la variación temporal y espacial de algunas especies dominantes de pomacentridos y labridos. Nuestros resultados sugieren una respuesta positiva a la protección de los peces de la Reserva Marina Playa Blanca, pero más estudios son necesarios para determinar el papel de las reservas marinas pequeñas en la conservación de la biodiversidad marina tropical.

Palabras clave: diversidad, estructura poblacional de peces, ictiofauna, Pacífico Este Tropical, arrecife rocoso, inspección visual, reservas marinas de pequeña escala, playas rocosas tropicales.

\section{REFERENCES}

Alvarez-Filip, L., H. Reyes-Bonilla \& L.E. CalderónAguilera. 2006. Community structure of fishes in Cabo Pulmo Reef, Gulf of California. Mar. Ecol. 27: 253-262.

Arburto-Oropeza, O. \& E. Balart. 2001. Community structure of reef fish in several habitats of a rocky reef in the Gulf of California. Mar. Ecol. 22: 283-305.

Campos, J.A., B. Burgos \& C. Gamboa. 1984. Effect of shrimp trawling on the commercial ichthyofauna of the Gulf of Nicoya, Costa Rica. Rev. Biol. Trop. 32: 203-207.

Castellanos-Galindo, G.A., A. Giraldo \& E.A. Rubio. 2005. Community structure of an assemblage of tidepool fishes on a Tropical Eastern Pacific rocky shore, Colombia. J. Fish Biol. 67: 392-408.

Castillo-Muñoz, R. 1983. Geology, p. 47-65. In D.H. Janzen (ed.). Costa Rican Natural History. University of Chicago, Chicago, USA.

Cortés, J. 1997. Biology and geology of eastern Pacific coral reefs. Coral Reefs 16: S39-S46.

Dominici-Arosemena, A. \& M. Wolff. 2006. Reef fish community structure in the Tropical Eastern Pacific (Panamá): living on a relatively stable rocky reef environment. Helgo. Mar. Res. 60: 287-305.
Dominici-Arosemena, A., E. Brugnoli-Olivera, J. CortésNúñez, J. Molina-Ureña \& M. Quesada-Alpizar. 2005. Community structure of eastern Pacific reef fishes (Gulf of Papagayo, Costa Rica). Tecnociencia 7: 19-41.

Edgar, G.J., S. Banks, J.M. Faina, M. Calvopina \& C. Martínez. 2004. Regional biogeography of shallow reef fish and macro-invertebrate communities in the Galapagos archipelago. J. Biogeogr. 31: 1107-1124.

Ferreira, C.E.L., J.E.A. Gonçalves \& R. Coutinho. 2001. Community structure of fishes and habitat complexity on a tropical rocky shore. Env. Biol. Fishes 61: 353-369.

Ferreira, C.E.L., S.R. Floeter, J.L. Gasparini, B.P. Ferreira \& J.C. Joyeux. 2004. Trophic structure patterns of Brazilian reef fishes: a latitudinal comparison. J. Biogeogr. 31: 1093-1106.

Floeter, S.R., C.E.L. Ferreira, A. Dominici-Arosemena \& I.R. Zalmon. 2004. Latitudinal gradients in Atlantic reef fish communities: trophic structure and spatial use patterns. J. Fish Biol. 64: 1680-1699.

Halpern, B.S. 2003. The impact of marine reserves: do reserves work and does reserve size matter? Ecol. Appl. 13: S117-S137

Halpern, B.S. \& R.R. Warner. 2002. Marine reserves have rapid and lasting effects. Ecol. Lett. 5: 361-366.

Hammer, Ø., D.A.T. Harper \& P.D. Ryan. 2001. PAST: Paleontological Statistics Software Package for Education and Data Analysis. Palaeontologia Electronica 4: 1-9.

Lubchenco, J., S.R. Palumbi, S.D. Gaines \& S. Andelman. 2003. Plugging a hole in the ocean: the emerging science of marine reserves. Ecol. Appl. 13: S3-S7.

Micheli, F., B.S. Halpern, L.W. Botsford \& R.R. Warner. 2004. Trajectories and correlates of community change in no-take marine reserves. Ecol. Appl. 14: 1709-1723.

National Research Council. NRC. 2001. Marine protected areas: tools for sustaining ocean ecosystems. National Academy, Washington D.C., USA.

Robertson, D.R. 1998. Do coral-reef fish faunas have a distinctive taxonomic structure? Coral Reefs 17: 179-186. 
Sobel, J. \& C. Dahlgren. 2004. Marine Reserves: A Guide to Science, Design, and Use. Island, Washington D.C., USA.

SYSTAT. 2010. SYSTAT version 12.0 for Windows. SYSTAT Software, Chicago, Illinois, USA.
Vroom, P.S., K.N. Page, J.C. Kenyon \& R.E. Brainard. 2006. Algae-dominated reefs. Amer. Sci. 94: 430-437.

Williams, D.M.B. 1982. Patterns in the distribution of fish communities across the Central Great Barrier Reef. Coral Reefs 1: 35-43. 